\title{
MONOTONICITY IN LAG FOR NON-MONOTONE MARKOV CHAINS
}

\author{
Rafał Kulik \\ Mathematical Institute \\ University of Wrocław \\ E-mail:rkuli@math.uni.wroc.pl \\ and \\ Department of Mathematics and Statistics \\ University of Ottawa \\ 585 King Edward Av. \\ K1N 6N5 Ottawa, ON, \\ Canada \\ E-mail: Rafal.Kulik@science.uottawa.ca
}

March 12, 2004

To appear in Probability in Engineering and Information Sciences

Keywords: monotonicity in lag; Markov chains; supermodular ordering;

Short title: Monotonicity in lag for Markov chains

AMS 2000 Subject Classification: 60J10, 60E15

\begin{abstract}
It is well known that for a stochastically monotone Markov chain $\left\{J_{n}\right\}_{n \geq 1}$ a function $\gamma(n)=\operatorname{Cov}\left[f\left(J_{1}\right), g\left(J_{n}\right)\right]$ is decreasing if $f$ and $g$ are increasing. We prove this property for a special subclass of non-monotone double stochastic Markov chains.
\end{abstract}

\section{Introduction}

Since the Daley's [2] paper it is well known that stochastically monotone Markov chain $\left\{J_{n}\right\}_{n \geq 1}$ has the following property. Let $f: \mathbb{R} \rightarrow \mathbb{R}_{+}$be monotone and $\gamma(n)=\operatorname{Cov}\left[f\left(J_{1}\right), f\left(J_{n}\right)\right]$. Then a map $n \rightarrow \gamma(n)$ is decreasing. Recall that a real-valued homogeneous discrete time Markov chain $\left\{J_{n}\right\}_{n \geq 1}$ is stochastically monotone, if its one step transition probability function $P\left(J_{n+1}>x \mid J_{n}=y\right)$ is non-decreasing in $x$ for every fixed $y$. Daley's result was extended in Bergmann and Stoyan [1]. Hu and Joe [3], (see also Joe [5], Theorems 8.3, 8.4, 8.7) stated conditions for the concordance ordering of bivariates of Markov chains under some monotonicity assumptions. The most general result was given in $\mathrm{Hu}$ and $\mathrm{Pan}$ [4]. If both the Markov chain and its time-reversed counterpart are stochastically 
monotone then $\gamma\left(n_{1}, \ldots, n_{m}\right)=E\left[f\left(J_{n_{1}}, \ldots, J_{n_{m}}\right)\right]$ is decreasing in $\left(n_{1}, \ldots, n_{m}\right)$ coordinatewise for each $m \geq 2$ and supermodular $f$. We refer to Müller and Stoyan [10] for a review of a recent work in this area. The monotonicity of a Markov chain is also sufficient for association of it (Lindqvist [7]). However, monotonicity is not necessary, see the Lindqvist's paper for a counterexample.

In our note we define a family of stationary and homogeneous Markov chains which are not necessary monotone. For that family we derive monotonicity of bivariates w.r.t. supermodular order and hence monotonicity of covariances. Therefore, we show that an answer to a question which appears in [9] is negative.

The paper is organized as follows. In Section 2 we collect some needed definitions and preliminary results. In Section 3 we define the family of Markov chains and prove the main result. Section 4 is devoted to examples and counterexamples. Some additional comparison results for our class are given in Section 5 .

\section{Preliminaries}

Define for $1 \leq i \leq m$ and $\varepsilon>0$ a difference operator $\Delta_{i}^{\varepsilon}$ by

$$
\Delta_{i}^{\varepsilon} \varphi\left(u_{1}, \ldots, u_{m}\right)=\varphi\left(u_{1}, \ldots, u_{i-1}, u_{i}+\varepsilon, u_{i+1}, \ldots, u_{m}\right)-\varphi\left(u_{1}, \ldots, u_{n}\right)
$$

for given $u_{1}, \ldots, u_{m}$. A function $\varphi: \mathbb{R}^{m} \rightarrow \mathbb{R}$ is called supermodular if for all $1 \leq i<j \leq m$ and $\varepsilon_{i}, \varepsilon_{j}>0$,

$$
\Delta_{i}^{\varepsilon_{i}} \Delta_{j}^{\varepsilon_{j}} \varphi(\mathbf{u}) \geq 0
$$

for all $\mathbf{u}=\left(u_{1}, \ldots, u_{m}\right)$. For smooth functions the above condition is equivalent to

$$
\frac{d^{2}}{d u_{i} d u_{j}} \varphi(\mathbf{u}) \geq 0
$$

for all $1 \leq i<j \leq n$. The standard examples of supermodular functions are: $u_{1} \times \cdots \times u_{m},-\max \left(u_{1}, \ldots, u_{m}\right), \min \left(u_{1}, \ldots, u_{m}\right), h\left(u_{1}+\cdots+u_{m}\right)$, where $h:$ $\mathbb{R} \rightarrow \mathbb{R}$ is convex.

This class of functions induce so called supermodular order. For arbitrary random vectors $\left(Y_{1}, \ldots, Y_{m}\right),\left(\tilde{Y}_{1}, \ldots, \tilde{Y}_{m}\right)$ we write

$$
\left(Y_{1}, \ldots, Y_{m}\right)<_{\mathrm{sm}}\left(\tilde{Y}_{1}, \ldots, \tilde{Y}_{m}\right)
$$

if $\mathbb{E}\left[\varphi\left(Y_{1}, \ldots, Y_{m}\right)\right] \leq \mathbb{E}\left[\varphi\left(\tilde{Y}_{1}, \ldots, \tilde{Y}_{m}\right)\right]$ for all supermodular $\varphi$ such that the respective expectations are finite. Similarly, for stationary random sequences $\left\{Y_{n}\right\}_{n \geq 1}$ and $\left\{\tilde{Y}_{n}\right\}_{n \geq 1}$ we write $\left\{Y_{n}\right\}<_{\mathrm{sm}}\left\{\tilde{Y}_{n}\right\}$ if for all $m \geq 1$,

$$
\left(Y_{1}, \ldots, Y_{m}\right)<_{\mathrm{sm}}\left(\tilde{Y}_{1}, \ldots, \tilde{Y}_{m}\right)
$$

Supermodular ordering is a dependence ordering in the sense of Joe [5]. In particularly, if $\left(Y_{1}, \ldots, Y_{m}\right)<_{\mathrm{sm}}\left(\tilde{Y}_{1}, \ldots, \tilde{Y}_{m}\right)$ then

- $Y_{i} \stackrel{\mathrm{d}}{=} \tilde{Y}_{i}$ for all $i=1, \ldots, m$, 
- $\operatorname{Cov}\left[Y_{i}, Y_{j}\right] \leq \operatorname{Cov}\left[\tilde{Y}_{i}, \tilde{Y}_{j}\right]$ for all $i, j=1, \ldots, m$.

Consider now two stationary homogeneous Markov chains $\left\{J_{n}\right\}_{n \geq 1},\left\{\tilde{J}_{n}\right\}_{n \geq 1}$ with the state space $\{1, \ldots, N\}$, the same stationary distribution $\pi=\left(\pi_{1}, \ldots, \pi_{N}\right)$ and transition matrices $\mathbf{P}=\left(p_{i j}\right)_{i, j=1}^{N}, \tilde{\mathbf{P}}=\left(\tilde{p}_{i j}\right)_{i, j=1}^{N}$, respectively. We shall write

$$
\mathbf{P}<_{\mathrm{sm}} \tilde{\mathbf{P}}
$$

if $\left(J_{1}, J_{2}\right)<_{\mathrm{sm}}\left(\tilde{J}_{1}, \tilde{J}_{2}\right)$. Here in the sequel we shall assume that all matrices have dimension $N \times N$.

For the supermodular ordering of $\left(J_{1}, J_{2}\right)$ and $\left(\tilde{J}_{1}, \tilde{J}_{2}\right)$ we have the following characterization (cf. Hu and Pan [4]).

Lemma 2.1 Let $\boldsymbol{\Pi}$ be a diagonal matrix $\operatorname{diag}\left(\pi_{1}, \ldots, \pi_{N}\right)$. Then

$$
\left(J_{1}, J_{2}\right)<_{\mathrm{sm}}\left(\tilde{J}_{1}, \tilde{J}_{2}\right)
$$

if and only if

$$
\sum_{i=1}^{r} \sum_{j=1}^{s}(\mathbf{\Pi P})_{i j} \leq \sum_{i=1}^{r} \sum_{j=1}^{s}(\boldsymbol{\Pi} \tilde{\mathbf{P}})_{i j}
$$

for all $r, s \in\{1, \ldots, N\}$.

The condition (2.2) is equivalent to a concordance ordering of bivariates, i.e.

$$
P\left(J_{1} \leq r, J_{2} \leq s\right) \leq P\left(\tilde{J}_{1} \leq r, \tilde{J}_{2} \leq s\right)
$$

for every $r, s$ in the state space. Note that for the double stochastic matrices $\mathbf{P}$ and $\tilde{\mathbf{P}}$ the condition (2.2) is equivalent to

$$
\sum_{i=1}^{r} \sum_{j=1}^{s} p_{i j} \leq \sum_{i=1}^{r} \sum_{j=1}^{s} \tilde{p}_{i j} .
$$

Moreover, supermodular ordering for the double stochastic matrices can be characterized in the following way. Let

$$
\mathbf{T}=\left[\begin{array}{ccccc}
1 & 0 & 0 & 0 & \cdots \\
1 & 1 & 0 & 0 & \cdots \\
1 & 1 & 1 & 0 & \cdots \\
\vdots & \vdots & \vdots & \vdots & \ddots
\end{array}\right]
$$

Note that

$$
\mathbf{T}^{t}=\left[\begin{array}{ccccc}
1 & 1 & 1 & 1 & \cdots \\
0 & 1 & 1 & 1 & \cdots \\
0 & 0 & 1 & 1 & \cdots \\
\vdots & \vdots & \vdots & \vdots & \ddots
\end{array}\right], \quad \mathbf{T}^{-1}=\left[\begin{array}{ccccc}
1 & 0 & 0 & 0 & \cdots \\
-1 & 1 & 0 & 0 & \cdots \\
0 & -1 & 1 & 0 & \cdots \\
\vdots & \vdots & \vdots & \vdots & \ddots
\end{array}\right] .
$$

The matrix $\mathbf{T}$ was used in Keilson and Kester [6] for obtaining stochastic monotonicity. A matrix $\mathbf{A}$ is stochastic monotone if and only if $\mathbf{T}^{-1} \mathbf{A T} \geq \mathbf{0}$, where $\mathbf{0}$ is a matrix which consists of zeros. 
Lemma 2.2 Assume that $\mathbf{P}$ and $\tilde{\mathbf{P}}$ are double stochastic. Then $\mathbf{P}<_{\mathrm{sm}} \tilde{\mathbf{P}}$ if and only if $\mathbf{T}(\tilde{\mathbf{P}}-\mathbf{P}) \mathbf{T}^{t} \geq \mathbf{0}$.

Proof. Inequality $\mathbf{T}(\tilde{\mathbf{P}}-\mathbf{P}) \mathbf{T}^{t} \geq \mathbf{0}$ is equivalent to $\sum_{i=1}^{r} \sum_{j=1}^{s} \tilde{p}_{i j} \geq \sum_{i=1}^{r} \sum_{j=1}^{s} p_{i j}$ for all $r$ and $s$, which means $\mathbf{P}<_{\text {sm }} \tilde{\mathbf{P}}$ by Eq. (2.3).

\section{Main result}

We introduce a class of Markov chains which are not necessary monotone.

Definition 3.1 We say that a matrix $\mathbf{A}$ belongs to a class $\mathcal{P S}$ if it is stochastic and $\mathbf{T A T}^{-1} \geq \mathbf{0}$. Equivalently, we say that a stationary homogeneous Markov chain belongs to $\mathcal{P S}$ if its transition probability matrix does.

Let $\mathbf{a}$ and $\mathbf{b}$ be $N$-dimensional vectors. We say that $\mathbf{a}$ precedes $\mathbf{b}$ in the partial sum ordering $\left(\mathbf{a} \prec_{p s} \mathbf{b}\right)$ if $\sum_{i=1}^{k} a_{i} \leq \sum_{i=1}^{k} b_{i}, k=1, \ldots, N$. Now, denote by $\mathbf{a}_{i}$, $i=1, \ldots, N$, the columns of a matrix $\mathbf{A}$. Then $\mathbf{A} \in \mathcal{P} \mathcal{S}$ if and only if

$$
\mathbf{a} \cdot \mathbf{N} \prec_{p s} \mathbf{a} \cdot \mathbf{N}-\mathbf{1} \prec_{p s} \cdots \prec_{p s} \mathbf{a} \cdot \mathbf{1} .
$$

Denote now by $\mathcal{D S}$ a class of double stochastic matrices. It turns out that for the Markov chains driven by $\mathbf{P} \in \mathcal{P S} \cap \mathcal{D S}$ the following monotonicity property holds.

Theorem 3.1 Let $\left\{J_{n}\right\}_{n \geq 1}$ be a stationary homogeneous Markov chain with a transition probability matrix $\mathbf{P} \in \mathcal{P S} \cap \mathcal{D S}$. Then for any $n \geq 1$,

$$
\left(J_{1}, J_{n+1}\right)<_{\mathrm{sm}}\left(J_{1}, J_{n}\right) .
$$

Corollary 3.1 Under conditions of Theorem 3.1 we have for the functions $f$ and $g$ both either nonincreasing or nondecreasing,

$$
\operatorname{Cov}\left[f\left(J_{0}\right), g\left(J_{n+1}\right)\right] \leq \operatorname{Cov}\left[f\left(J_{0}\right), g\left(J_{n}\right)\right] .
$$

The proof of Theorem 3.1 consists of the sequence of Lemmas.

Lemma 3.1 1. If $\mathbf{A}, \mathbf{B} \in \mathcal{P S}$ then $\mathbf{A B} \in \mathcal{P S}$;

2. If $\mathbf{A}(i) \in \mathcal{P} \mathcal{S}, i=1, \ldots, k$ and $\mathbf{w}=\left(w_{1}, \ldots, w_{k}\right)$ is a probability vector then $\sum_{i=1}^{k} w_{i} \mathbf{A}(i) \in \mathcal{P S}$.

Proof.

1. We have $\mathbf{T A B} \mathbf{T}^{-1}=\mathbf{T A} \mathbf{T}^{-1} \mathbf{T B} \mathbf{T}^{-1} \geq \mathbf{0}$ from the assumptions on $\mathbf{A}$ and B.

2. Obvious. 
Lemma 3.2 Assume that $\mathbf{B}, \tilde{\mathbf{B}} \in \mathcal{D S}$ and $\mathbf{A} \in \mathcal{P S}$. If $\mathbf{B}<_{\mathrm{sm}} \tilde{\mathbf{B}}$ then $\mathbf{A B}<_{\mathrm{sm}}$ $\mathbf{A} \tilde{\mathbf{B}}$ and $\mathbf{B} \mathbf{A}<_{\text {sm }} \tilde{\mathbf{B}} \mathbf{A}$.

Proof. We have

$$
\begin{aligned}
\mathbf{T}(\mathbf{A} \tilde{\mathbf{B}}-\mathbf{A B}) \mathbf{T}^{t} & =\mathbf{T A}(\tilde{\mathbf{B}}-\mathbf{B}) \mathbf{T}^{t} \\
& =\left[\mathbf{T A} \mathbf{T}^{-1}\right]\left[\mathbf{T}(\tilde{\mathbf{B}}-\mathbf{B}) \mathbf{T}^{t}\right]
\end{aligned}
$$

The first term in brackets is greater than $\mathbf{0}$ due to assumptions on $\mathbf{A}$, the second one has the same property because of Lemma 2.2. Therefore $\mathbf{T}(\mathbf{A} \tilde{\mathbf{B}}-\mathbf{A B}) \mathbf{T}^{t} \geq \mathbf{0}$ and using Lemma 2.2 once more we obtained the comparison result.

Lemma 3.3 Assume that $\mathbf{A} \in \mathcal{P} \mathcal{S} \cap \mathcal{D S}$. Then $\mathbf{A}^{2}<_{\text {sm }} \mathbf{A}$. Moreover, for every $n \geq 1, \mathbf{A}^{n+1}<_{\mathrm{sm}} \mathbf{A}^{n}$.

Proof. Note that for any double stochastic matrix $\mathbf{A}$ we have $\mathbf{A}<_{\text {sm }} \mathbf{I}$, where $\mathbf{I}$ is an identity matrix. Moreover, $\mathbf{I} \in \mathcal{D S}$. From Lemma 3.2 we have $\mathbf{A}^{2}<_{\text {sm }} \mathbf{A}$. Assume now that $\mathbf{A}^{n}<_{\mathrm{sm}} \mathbf{A}^{n-1}$. Because of Lemma $3.1 \mathbf{A}^{n}$ and $\mathbf{A}^{n-1}$ belong to $\mathcal{P S}$. Moreover, they are double stochastic. Therefore, Lemma 3.2 applies and we have $\mathbf{A} \mathbf{A}^{n}<_{\text {sm }} \mathbf{A} \mathbf{A}^{n-1}$.

Proof of the Theorem 3.1. Denote by $\mathbf{P}^{(k)}=\left(p_{i j}^{(k)}\right)_{i, j=1}^{N}, k \geq 1$, the $k$-step probability matrix for $\left\{J_{n}\right\}_{n \geq 1}$. From Lemma 3.3 we have that

$$
\sum_{i=1}^{r} \sum_{i=1}^{s} p_{i j}^{(n+1)} \leq \sum_{i=1}^{r} \sum_{i=1}^{s} p_{i j}^{(n)}
$$

for all $r, s=1, \ldots, N$. Therefore, $\left(J_{1}, J_{n+1}\right)<_{\mathrm{sm}}\left(J_{1}, J_{n}\right)$.

The Corollary 3.1 follows from stationarity of a Markov chain and the fact that for the functions $f$ and $g$ both either nonincreasing or nondecreasing the function $\varphi(x, y)=f(x) g(y)$ is supermodular.

\section{Examples and Counterexamples}

Example 4.1 Let $p$ and $\varepsilon>0$ be such that $p \geq 2 \varepsilon$ and $p+\varepsilon \leq 1$. Then the following matrix belongs to $\mathcal{P S} \cap \mathcal{D S}$ and is not monotone.

$$
\mathbf{P}=\frac{1}{4 p-2 \varepsilon}\left[\begin{array}{cccc}
p+\varepsilon & p+\varepsilon & p-2 \varepsilon & p-2 \varepsilon \\
p-\varepsilon & p-\varepsilon & p & p \\
p & p & p-\varepsilon & p-\varepsilon \\
p-2 \varepsilon & p-2 \varepsilon & p+\varepsilon & p+\varepsilon
\end{array}\right]
$$


Example 4.2 The lemma 3.3 fails if one of the assumptions is removed. Let

$$
\mathbf{A}=\frac{1}{36}\left[\begin{array}{cccc}
18 & 0 & 18 & 0 \\
6 & 12 & 6 & 12 \\
7 & 11 & 7 & 11 \\
5 & 13 & 5 & 13
\end{array}\right]
$$

Then $\mathbf{A} \in \mathcal{D} \mathcal{S}, \mathbf{A} \notin \mathcal{P} \mathcal{S}$ and it is not true that $\mathbf{A}^{2}<_{\text {sm }} \mathbf{A}$. The above matrix was used in Lindqvist [7] as a counterexample of a Markov chain which is associated but not stochastically monotone.

Let now

$$
\mathbf{A}=\frac{1}{4}\left[\begin{array}{llll}
1 & 1 & 1 & 1 \\
1 & 1 & 1 & 1 \\
2 & 1 & 1 & 0 \\
2 & 1 & 1 & 0
\end{array}\right]
$$

This matrix belongs to $\mathcal{P} \mathcal{S}$ but is neither double stochastic nor monotone. It is not true that $\mathbf{A}^{2}<_{\text {sm }} \mathbf{A}$.

\section{Additional comparison results}

Observe that for $a \leq a^{\prime}$ and stochastic matrices $\mathbf{A}, \mathbf{B}$ with the same invariant distributions we have $a^{\prime} \mathbf{A}+\left(1-a^{\prime}\right) \mathbf{B}<_{\mathrm{sm}} a \mathbf{A}+(1-a) \mathbf{B}$ if $\mathbf{A}<_{\mathrm{sm}} \mathbf{B}$. This can be interpreted as follows. Let $\left\{J_{n}\right\}_{n \geq 1},\left\{\tilde{J}_{n}\right\}_{n \geq 1}$ be the stationary homogeneous Markov chains with transition matrices $\mathbf{A}$ and $\mathbf{B}$, respectively. Define $Z_{n}=$ $\Theta J_{n}+(1-\Theta) \tilde{J}_{n}$ and $\tilde{Z}_{n}=\tilde{\Theta} J_{n}+(1-\tilde{\Theta}) \tilde{J}_{n}$, where $\Theta, \tilde{\Theta}$ are Bernoulli random variables with $P(\Theta=1)=a, P(\tilde{\Theta}=1)=a^{\prime}$, independent of everything else. Then $\left(\tilde{Z}_{1}, \tilde{Z}_{2}\right)<_{\mathrm{sm}}\left(Z_{1}, Z_{2}\right)$ provided $\left(J_{1}, J_{2}\right)<_{\mathrm{sm}}\left(\tilde{J}_{1}, \tilde{J}_{2}\right)$ and $a \leq a^{\prime}$. If we have some additional monotonicity properties we can have comparison of the whole sequences $\left\{Z_{n}\right\}_{n \geq 1}$ and $\left\{\tilde{Z}_{n}\right\}_{n \geq 1}$. However, the above consideration can not be rewritten for the random variables $\Theta, \tilde{\Theta}$ assuming their values in $\{1, \ldots, K\}$. This can be done if the transition matrices belong to $\mathcal{P S} \cap \mathcal{D S}$. We refer to Marshall and Olkin [8] for the concept of majorization.

Proposition 5.1 Assume that

(a) $\mathbf{P}(k):=\left(p_{i j}(k)\right)_{i, j=1}^{N} \in \mathcal{P} \mathcal{S} \cap \mathcal{D S}, k=1, \ldots, K$;

(b) $\mathbf{P}(1)<_{\mathrm{sm}} \mathbf{P}(2)<_{\mathrm{sm}} \cdots<_{\mathrm{sm}} \mathbf{P}(K)$;

(c) $\mathbf{a}$ is majorized by $\mathbf{b}$ (we write $\mathbf{a} \prec \mathbf{b}$ ), where $\mathbf{a}, \mathbf{b}$ are $K$-dimensional probability vectors with coordinates arranged in the decreasing order.

Then

$$
\mathbf{P}:=\sum_{k=1}^{K} b_{k} \mathbf{P}(k)<_{\text {sm }} \sum_{k=1}^{K} a_{k} \mathbf{P}(k)=: \tilde{\mathbf{P}}
$$


Proof. Define for $l=1, \ldots, N$ and $r=1, \ldots, K$ the vectors

$$
\mathbf{v}^{(l, r)}:=\left(v_{1}^{(l, r)}, \ldots, v_{N}^{(l, r)}\right),
$$

where for $j=1, \ldots, N, v_{j}^{(l, r)}$ are defined as

$$
v_{j}^{(l, r)}:=\sum_{i=1}^{l} p_{i j}(r) .
$$

According to assumption (a) these vectors have decreasing coordinates. Moreover, because of (b) we have

$$
\mathbf{v}^{(l, 1)} \prec \mathbf{v}^{(l, 2)} \prec \cdots \prec \mathbf{v}^{(l, K)}
$$

for each fixed $l$. Take now

$$
\mathbf{w}^{l}:=\sum_{r=1}^{K} b_{r} \mathbf{v}^{(l, r)}
$$

and

$$
\tilde{\mathbf{w}}^{l}:=\sum_{r=1}^{K} a_{r} \mathbf{v}^{(l, r)} .
$$

From Marshall and Olkin [8, p. 125] we have $\mathbf{w}^{l} \prec \tilde{\mathbf{w}}^{l}$ for every $l=1, \ldots, N$. Because $\mathbf{w}^{l}$ and $\tilde{\mathbf{w}}^{l}$ have decreasing coordinates majorization order implies $\mathbf{w}^{l} \prec_{p s}$ $\tilde{\mathbf{w}}^{l}$. Observe now that $j$ th coordinates of $\mathbf{w}^{l}$ and $\tilde{\mathbf{w}}^{l}$ can be written as

$$
w^{l}(j)=\sum_{i=1}^{l} p_{i j}
$$

and

$$
\tilde{w}^{l}(j)=\sum_{i=1}^{l} \tilde{p}_{i j},
$$

respectively. It implies comparison result.

Acknowledgement The author is grateful to Ryszard Szekli for many helpful comments. This work was partially done during the author's stay at University of Ottawa.

\section{References}

[1] Bergmann, R. \& Stoyan, D. (1978). Monotonicity properties of second order characteristics of stochastically monotone Markov chains. Mathematische Nachrichten 82: 99-102.

[2] Daley, D.J. (1968). Stochastically monotone Markov chains. Zeitschrift fr Wahrscheinlichkeitstheorie und Verwandte Gebiete 10: 305-317. 
[3] Hu, T. \& Joe, H. (1995). Monotonicity of positive dependence with time for stationary reversible Markov chains. Probability in the Engineering and Informational Sciences 9: 227-237.

[4] Hu, T. \& Pan, X. (2000). Comparisons of dependence for stationary Markov processes. Probability in the Engineering and Informational Sciences 14: 299-315.

[5] Joe, H. (1997). Multivariate models and dependence concepts. London: Chapman \& Hall.

[6] Keilson, J. \& Kester, A. (1977). Monotone matrices and monotone Markov processes. Stochastic Processes and their Applications 5: 231-241.

[7] Lindqvist, B.H. (1987). Monotone and associated Markov chains, with applications to reliability theory. Journal of Applied Probability 24: 679-695.

[8] Marshall, A.W. \& Olkin, I. (1979). Inequalities: Theory of Majorization and Its Applications. Academic Press.

[9] Miyoshi, N. \& Rolski, T. (2003). Ross type conjectures on monotonicity of queues. Australian and New Zealand Journal of Statistics, to appear.

[10] Müller A. \& Stoyan, D. (2002). Comparison Methods for Stochastic Models and Risks. New York: Wiley. 